Review began 11/09/2021 Review ended 11/13/2021 Published 11/15/2021

๑) Copyright 2021

Hind et al. This is an open access article distributed under the terms of the Creative Commons Attribution License CC-BY 4.0., which permits unrestricted use, distribution, and reproduction in any medium, provided the original author and source are credited.

\section{Myositis, Osteomyelitis and a Parasymphyseal Stress Fracture in a Paediatric Patient}

\author{
Jamie Hind ${ }^{1}$, Aimen Gmati ${ }^{1}$, Neil Ashwood ${ }^{2}$ \\ 1. Trauma and Orthopaedics, Oxford University Hospitals National Health Service Foundation Trust, Oxford, GBR 2. \\ Trauma and Orthopaedics, University Hospitals of Derby and Burton National Health Service Foundation Trust, \\ Burton, GBR \\ Corresponding author: Aimen Gmati, aimengmati@gmail.com
}

\begin{abstract}
The limping child and painful hip are common presentations in many paediatric emergency units. Typically caused by mild self-limiting events, less commonly, they may be implicated in one of a group of inflammatory myopathies, or myositis. Diagnosis of this condition can be extremely difficult, and is aided by thorough clinical assessment, radiological imaging, and extensive blood serum testing. Myositis with associated osteomyelitis and a pathological fracture is an incredibly rare finding, described in this case report in a seven-year-old child.
\end{abstract}

Categories: Pediatrics, Orthopedics, Rheumatology

Keywords: dermatomyositis, anti-jo-1 antibodies, immune myositis, parasymphysis fractures, osteomyelitis diagnosis, inflammatory myositis

\section{Introduction}

Inflammatory disorders of the skeletal system are a group of rare diseases that show chronic muscular inflammation, and less often, extra-skeletal manifestations. Numerous classifications of myositis exist, determined by a careful assessment of clinical symptoms, radiological imaging and a close analysis of associated auto-antibodies [1]. Osteomyelitis is an inflammatory reaction in skeletal muscle, usually associated with bacteria, namely Staphylococcus as the main causative agent [2]. The combination of myositis, osteomyelitis and a stress fracture has not been documented in previous literature.

\section{Case Presentation}

A seven-year old boy was seen in the emergency department with a temperature and a painful right hip. This was thought to be a result of viral arthritis or an irritable hip. The patient was discharged home and his symptoms resolved. The patient was referred to the orthopaedic team after his symptoms returned, causing him significant pain to his right hip and preventing him from attending school. Apart from eczema, he had no other medical issues. On examination he had a very slight restriction of internal rotation in the right hip when compared to the left. However, this was completely pain free. A plain radiograph of the pelvis was performed, which was normal with no evidence of bony injury or Perthes disease (Figure 1). 


\section{Cureus}

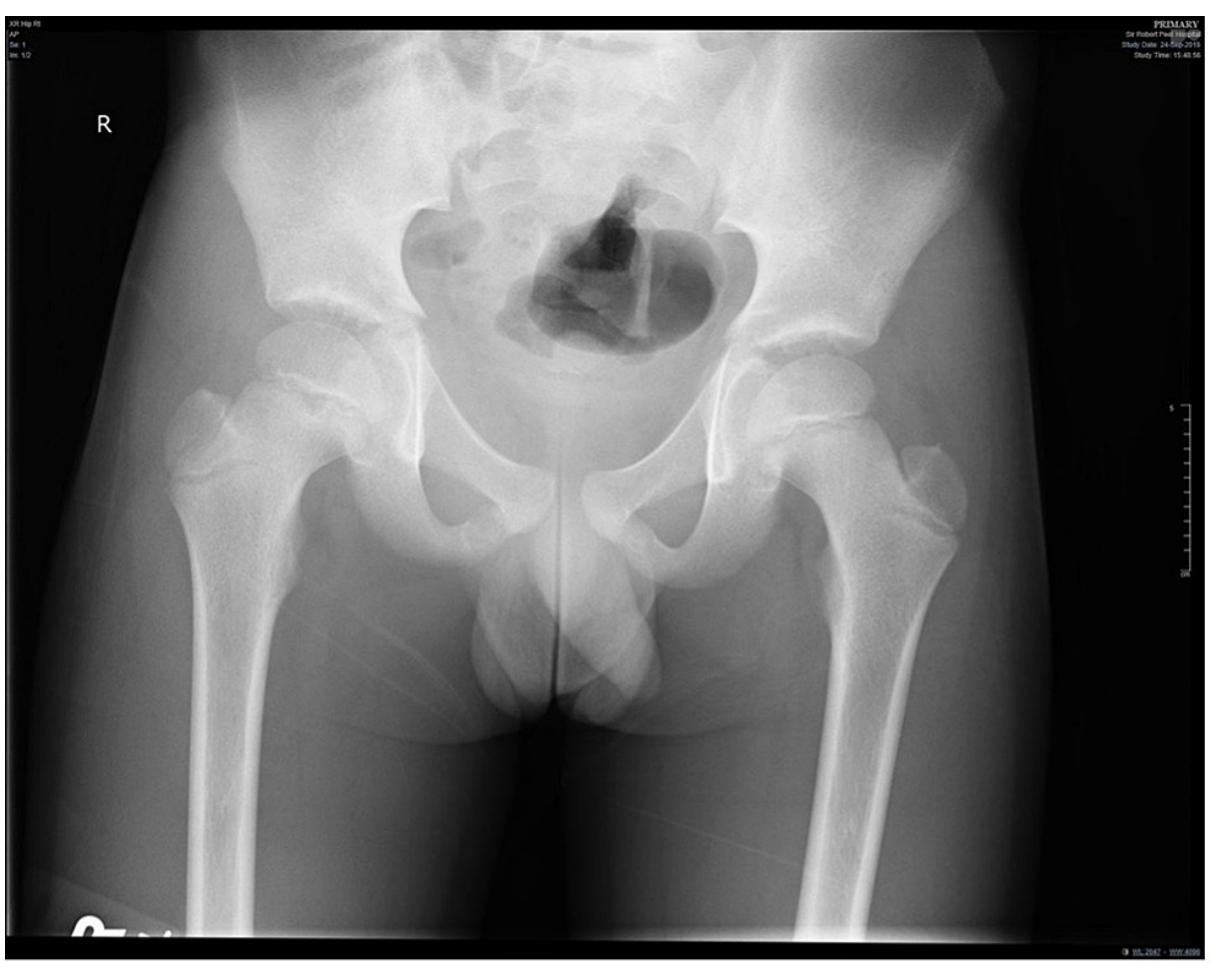

\section{FIGURE 1: Anteroposterior pelvic radiograph showing normal pelvic anatomy, normal growth plates, and no evidence of bony injury or Perthes disease.}

An MRI was requested but before this was performed the patient had another episode of right hip pain and a temperature of 38.2. He was, therefore, referred again to the orthopaedic team. On this occasion he walked into clinic with an antalgic gait. On examination he had some limitation of internal rotation compared to the left side. He had pain and tenderness around the right hip joint. He had normal neurovascular status distally. He had his MRI scan, which showed extensive myositis of the right obturator externus muscle and pectineus muscle with a small pocket of high signal collection (Figure 2). It also demonstrated osteomyelitis in the right pubic bone. He was seen in clinic and scan results explained to the patient and his family. At this time he was relatively comfortable. He had a full range of motion to the hip and there was no tenderness around the hip joint. He could straight leg raise without any problems. There were no reported spikes in temperature. He was eating, drinking and playing as normal. His white cell count and C-reactive protein were normal but erythrocyte sedimentation rate was raised slightly at 16 . 


\section{Cureus}

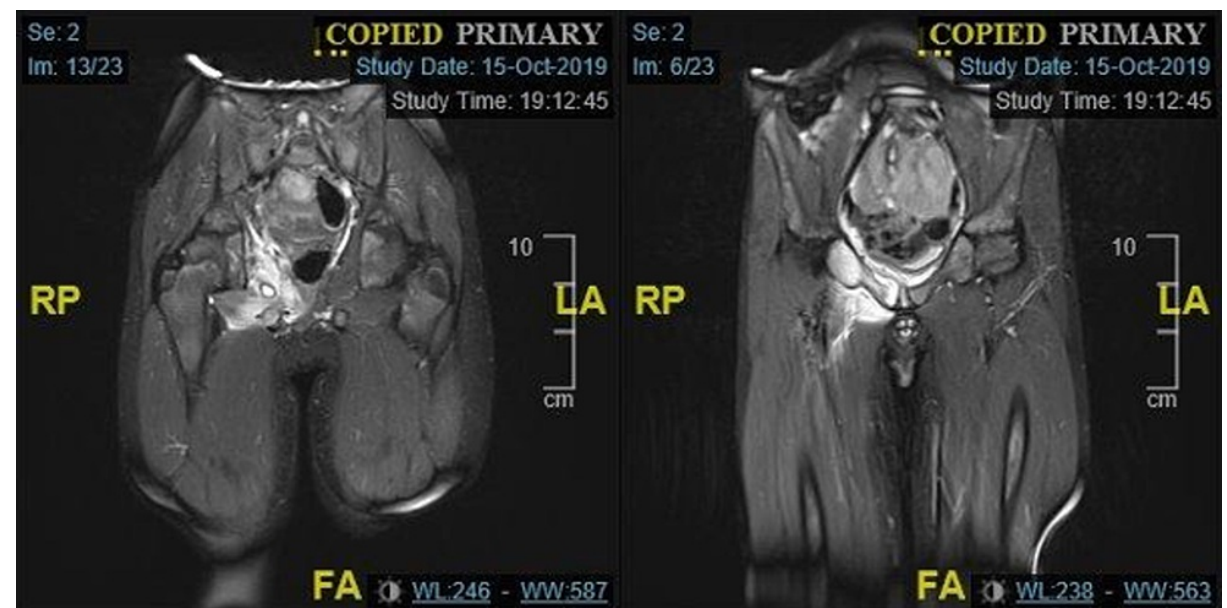

FIGURE 2: Coronal sections from T2-weighted MRI showing extensive myositis in the right obturator internus containing a collection, less severe myositis in the obturator externus and pectineus muscle (left) and suspicion of osteomyelitis of right pubic bone (right).

A second MRI scan was performed in December, which showed significant reduction in the degree of oedema at the right ischiopubic synchondrosis and in the surrounding soft tissue, particularly abductor and lateral rotators (Figure 3). There was still marked oedema in the right parasymphyseal region likely in keeping with a further area of stress reaction or stress fracture. A further MRI was performed six weeks later (Figure 4), which showed almost complete resolution of the abnormal high signal in the right obturator internus and pectineus and interval improvements in the inferior aspect of left sacral ala. There was, however, little interval change in the oedema/inflammation in the right superior and inferior pubis rami including the synchondrosis. The patient was referred to the paediatric orthopaedic consultant. At this point, clinically he was apyrexial and clinically well. He had a normal stance and a normal gait. He had normal power in both lower limbs and a full range of movement. He had no back or hip pain. Whilst it appears that this condition has improved and may resolve, we are unable to confirm the exact aetiology and the pathophysiology. 


\section{Cureus}

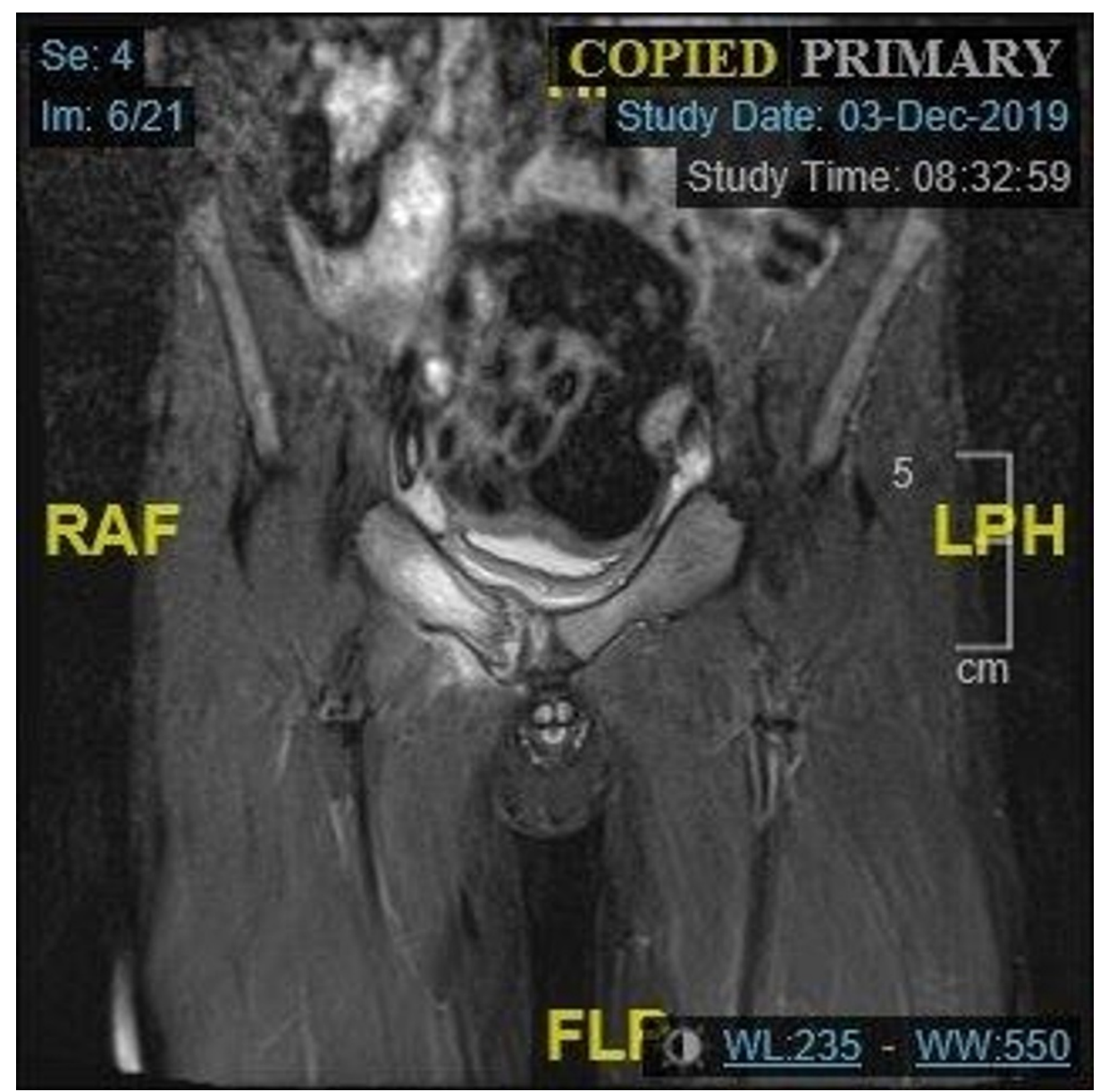

FIGURE 3: Coronal sections of T2-weighted MRI demonstrating marked oedema in the right parasymphyseal region, likely in keeping with a further area of stress reaction or stress fracture. 


\section{Cureus}

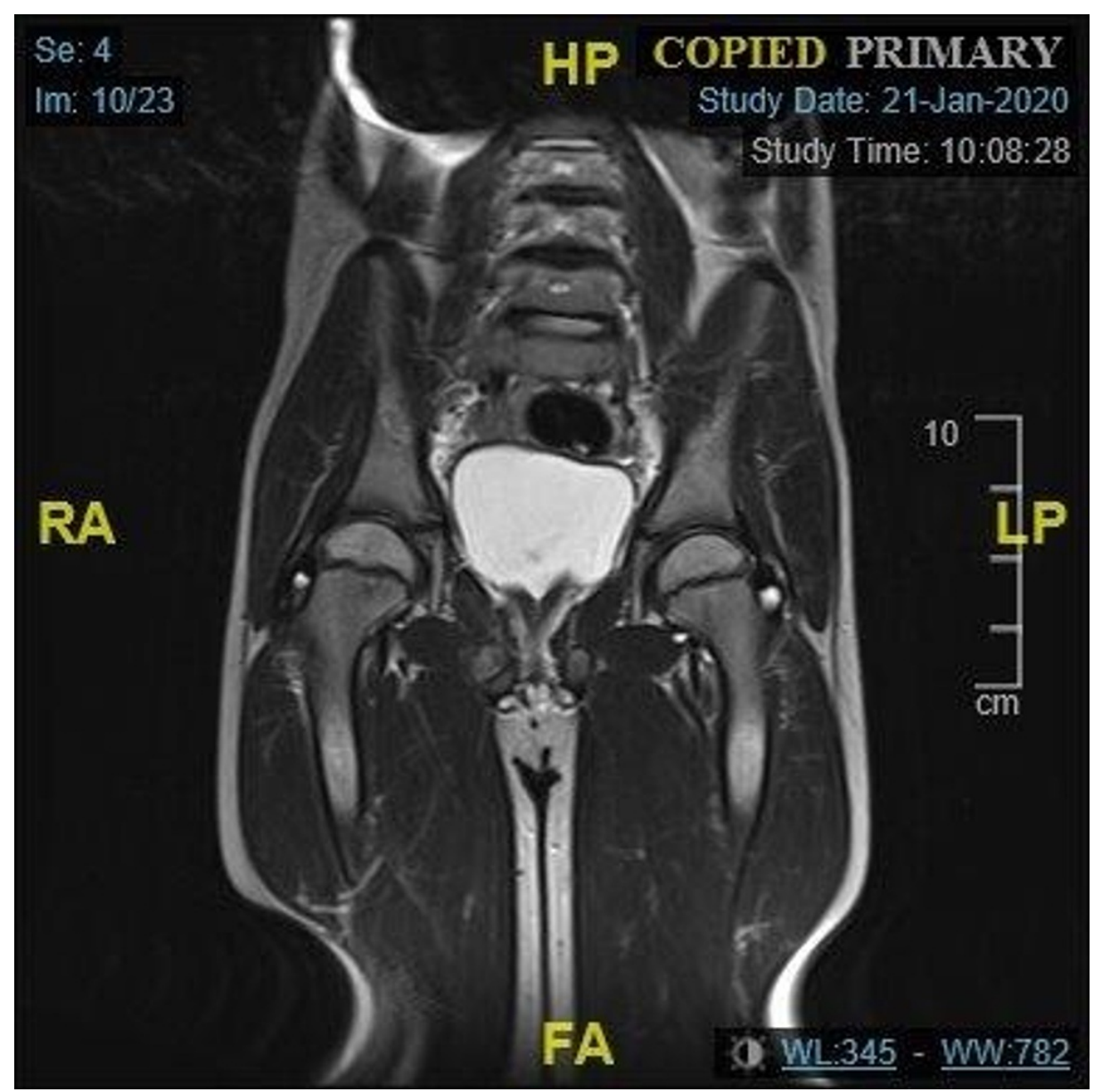

FIGURE 4: Coronal sections of T2-weighted MRI demonstrating almost complete resolution of the abnormal high signal in the right obturator internus and pectineus, and interval improvements in the inferior aspect of the left sacral ala.

\section{Discussion}

Autoimmune myositis, previously known as idiopathic inflammatory myopathies, is a group of rare heterogeneous disorders characterized by chronic inflammation of skeletal muscle leading to weakness of the affected muscle(s). It can also affect other organs including skin, lungs, heart and gastrointestinal tract; however, this is less common [3]. Originally, myositis was sub-classified into polymyositis, dermatomyositis (DM) and inclusion body myositis [3]. Now it also encompasses overlap myositis [4] and necrotizing autoimmune myositis [5]. DM is an autoimmune disease that primarily involves both skin and skeletal muscles and the cutaneous manifestation in these patients may be present up to two years before any evidence of myositis occurs [6]. This is the most common sub-type of myositis [7], and unlike the other types of myositis that affect middle-aged individuals, DM predominantly affects children and adults [8]. The cutaneous manifestations of DM are well documented and some of the more specific skin lesions include Gottrons papules, violaceous scaling papules on the skin overlying the joints and proximal nail folds; Gottrons sign, violaceous patches overlying the knees; ' $V$ ' sign, confluent macular erythema over the lower anterior neck and upper anterior chest; and the Shawl sign, erythema over the upper back, posterior neck, and shoulders and lateral arms [9]. Other skin manifestations include telangiectasia, erythema over the elbows and knees, dystrophic cuticles and calcinosis [10]. The above skin lesions may be worsened by exposure to ultraviolet (UV) A and UVB light [6]. Interstitial lung disease is an important associated condition in up to $40 \%$ patients with DM causing significant morbidity and mortality to these patients [11]. There is no set diagnostic criteria for myositis, so it is often a clinical diagnosis based on history, examination and investigation findings.

The history of DM is often of proximal muscle weakness initially, with or without myalgia or tenderness and the skin lesions as mentioned previously [12]. On examination the patient experiences pain when the affected muscles are stretched or contracted or the affected muscle is palpated. Blood test reveals elevated enzyme levels, most notably creatine kinase. MRI of the muscle and electromyography are also used in the work-up for patients with suspected myositis as well as detailed histopathological examination of a biopsy 
of the skeletal muscle [5]. Myositis-specific autoantibodies are detected to help diagnose myositis and also to help identify the clinical sub-group [13]. The Jo- 1 autoantibody is the most common [14]. Treatment is usually initiated with intravenous glucocorticosteroids, followed by oral corticosteroid with a tapering regime [15]. Our patient, however, did not receive any treatment. Myositis in combination with osteomyelitis is a rare phenomenon and has only been reported in a few case reports: One in a patient with a Pasteurella multocida infection [16], one in a patient following a course of radiotherapy [17], and one in a patient with chronic recurrent multifocal osteomyelitis [18]. This case is unlike the aforementioned, as no cause could be identified. Whilst it may be possible that his eczema predisposed him to myositis and osteomyelitis, equally, this may have been a cutaneous manifestation of myositis not previously documented. In addition to this, this case report is also unique in that the patient had an associated pathological stress fracture. The combination of myositis, osteomyelitis and an associated stress fracture has not been documented previously [19]. Whilst it has been noted that pathological stress fractures are a recognized but rare complication of osteomyelitis [20], its association with myositis is less well documented.

\section{Conclusions}

Autoimmune myositis is a rare condition associated with significant morbidity. DM is the most common subtype of this condition, especially in children. Myositis with associated osteomyelitis is a particularly rare finding. This case report is of a patient with an atypical presentation of myositis, with associated osteomyelitis and a pathological stress fracture.

\section{Additional Information \\ Disclosures}

Human subjects: Consent was obtained or waived by all participants in this study. Conflicts of interest: In compliance with the ICMJE uniform disclosure form, all authors declare the following: Payment/services info: All authors have declared that no financial support was received from any organization for the submitted work. Financial relationships: All authors have declared that they have no financial relationships at present or within the previous three years with any organizations that might have an interest in the submitted work. Other relationships: All authors have declared that there are no other relationships or activities that could appear to have influenced the submitted work.

\section{References}

1. Patient Education: Polymyositis, Dermatomyositis, and Other Forms of Idiopathic Inflammatory Myopathy (Beyond the Basics). (2020). Accessed: October 15, 2021: https://www.uptodate.com/contents/polymyositisdermatomyositis-and-other-forms-of-idiopathic-inflammatory-myopathy-be....

2. Fritz JM, McDonald JR: Osteomyelitis: approach to diagnosis and treatment. Phys Sportsmed. 2008, 36:nihpa116823. 10.3810/psm.2008.12.11

3. Lundberg IE, de Visser M, Werth VP: Classification of myositis. Nat Rev Rheumatol. 2018, 14:269-78. 10.1038/nrrheum.2018.41

4. Troyanov Y, Targoff IN, Tremblay JL, Goulet JR, Raymond Y, Senécal JL: Novel classification of idiopathic inflammatory myopathies based on overlap syndrome features and autoantibodies: analysis of 100 French Canadian patients. Medicine (Baltimore). 2005, 84:231-49. 10.1097/01.md.0000173991.74008.b0

5. Dalakas MC: Inflammatory muscle diseases. N Engl J Med. 2015, 372:1734-47. 10.1056/NEJMra1402225

6. Santmyire-Rosenberger B, Dugan EM: Skin involvement in dermatomyositis. Curr Opin Rheumatol. 2003, 15:714-22. 10.1097/00002281-200311000-00006

7. Lilleker JB, Vencovsky J, Wang G, et al.: The EuroMyositis registry: an international collaborative tool to facilitate myositis research. Ann Rheum Dis. 2018, 77:30-9. 10.1136/annrheumdis-2017-211868

8. Schmidt J: Current classification and management of inflammatory myopathies. J Neuromuscul Dis. 2018, 5:109-29. 10.3233/JND-180308

9. Marvi U, Chung L, Fiorentino DF: Clinical presentation and evaluation of dermatomyositis. Indian J Dermatol. 2012, 57:375-81. 10.4103/0019-5154.100486

10. Callen JP, Wortmann RL: Dermatomyositis. Clin Dermatol. 2006, 24:363-73. 10.1016/i.clindermatol.2006.07.001

11. Fathi M, Lundberg IE, Tornling G: Pulmonary complications of polymyositis and dermatomyositis. Semin Respir Crit Care Med. 2007, 28:451-8. 10.1055/s-2007-985666

12. Lundberg IE, Miller FW, Tjärnlund A, Bottai M: Diagnosis and classification of idiopathic inflammatory myopathies. J Intern Med. 2016, 280:39-51. 10.1111/joim.12524

13. Love LA, Leff RL, Fraser DD, Targoff IN, Dalakas M, Plotz PH, Miller FW: A new approach to the classification of idiopathic inflammatory myopathy: myositis-specific autoantibodies define useful homogeneous patient groups. Medicine (Baltimore). 1991, 70:360-74. 10.1097/00005792-199111000-00002

14. Mimori T, Nakashima R, Hosono Y: Interstitial lung disease in myositis: clinical subsets, biomarkers, and treatment. Curr Rheumatol Rep. 2012, 14:264-74. 10.1007/s11926-012-0246-6

15. Carstens PO, Schmidt J: Diagnosis, pathogenesis and treatment of myositis: recent advances . Clin Exp Immunol. 2014, 175:349-58. 10.1111/cei.12194

16. Marcantonio Y, Kulkarni P, Sachs S, Ting K, Lee J, Mendoza D: Disseminated Pasteurella multocida infection: cellulitis, osteomyelitis, and myositis. IDCases. 2017, 10:68-70. 10.1016/jidcr.2017.09.004

17. Borregón Rivilla M, Serrano Romero de Ávila V, Acevedo Ribó M, David Cárdenas J, Ignacio Chacón LópezMuñiz J, Esteban Esteban C: Myositis and osteomyelitis due to radiotherapy-related fistulae in locally 


\section{Cureus}

advanced cervical cancer. J Case Rep Images Obstet Gynecol. 2019, 5:100051Z08MR2019.

10.5348/100051Z08MR2019CS

18. Nagashima S, Nozawa T, Kizawa T, et al.: [Chronic recurrent multifocal osteomyelitis with interstitial myositis]. Nihon Rinsho Meneki Gakkai Kaishi. 2013, 36:52-7. 10.2177/jsci.36.52

19. Canella C, Costa F, Danowisk A, de Melo AS, Marchiori E: Stress fracture and osteomyelitis in a patient with systemic lupus erythematosus. Radiol Bras. 2018, 51:277-8. 10.1590/0100-3984.2017.0035

20. Walmsley BH: Pathological fracture in acute osteomyelitis in an adult . J R Soc Med. 1983, 76:77-8. 